\title{
A expansão das Universidades Federais e os seus efeitos de curto prazo sobre os Indicadores Municipais
}

\author{
The expansion of Federal Universities and \\ their short-term effects on Municipal Indicators
}

\author{
Mayara Lima Casqueiro ${ }^{1}$ \\ ${ }^{1}$ Universidade Federal do Ceará | Faculdade | Departamento | Fortaleza | CE | Brasil. Contato: \\ mayaralima@alu.ufc.br. ORCID: https://orcid.org/0000-0002-5899-2087
}

\section{Guilherme Irffi ${ }^{2}$}

${ }^{2}$ Universidade Federal do Ceará | Faculdade de Economia Administração, Atuária, Contabilidade e Secretariado e Executivo | Fortaleza | CE | Brasil. Contato: guidirffi@gmail.com. ORCID: https://orcid.org/0000-0002-3558-7628

\section{Cristiano da Costa da Silva ${ }^{3}$}

${ }^{3}$ Universidade do Estado do Rio Grande do Norte | Faculdade de Ciências Econômicas | Departamento de Economia | Mossoró | RN | Brasil. Contato: cristiano.dacostadasilva@ hotmail.com. ORCID: https://orcid.org/0000-0001-9534-5332

Resumo: Diante do quadro de expansão da rede de educação superior, este estudo se propõe a analisar o impacto da expansão das Universidades Federais sobre o desenvolvimento local dos municípios brasileiros, baseado em indicadores econômicos, sociais e de mercado de trabalho. Além disso, busca-se identificar os fatores que influenciaram os municípios a serem contemplados com um campus da Universidade. Para isso, emprega-se o método de pareamento com escore de propensão com objetivo de construir um grupo de controle que mais se assemelha ao grupo de tratamento. E, para estimar os efeitos, após o pareamento, são utilizados os estimadores de Diferenças em Diferenças e de Efeito Fixo. Os resultados indicam que municípios que possuem campus universitário particular, população superior a 50 mil habitantes e com maior relevância econômica (importância do PIB) na sua microrregião são fatores (ou características) que influenciaram na escolha dos municípios beneficiados com a política de expansão. Já em relação aos efeitos da expansão sobre os indicadores, observa-se que a criação de novos campi reduz o percentual de pobres nos municípios, em média, em 1,5 p.p. Além disso, há evidência (fraca) de que a política aumenta a renda per capita, o percentual de ocupados com nível superior e com nível médio. Contudo, vale ressaltar que talvez seja necessário maior período de tempo para que os efeitos da política de expansão causem impactos significativos sobre a economia.

Palavras-chave: Avaliação de impacto. Expansão das universidades federais. Indicadores Municipais.

Abstract: In the light of the expansion of the higher education network, the propose od this study is to analyze the impact of Federal Universities expansion on Brazilian cities development based on economic, social and labor market indicators. In addition to his, it was sought to identify the factors that influenced the municipalities to be contemplated with a university campus. Moreover, the propensity score matching method was used to construct a control group that most closely resembled a treatment group. And, to estimate the effects, after the pairing, the Differences in Differences and Fixed Effect estimators were used. The results indicate that municipalities with a private university campus, population of more than 50,000 inhabitants and with greater economic relevance (importance of GDP) in their micro-region are factors (or characteristics) that influenced the choice of municipalities benefited by the expansion policy. Regarding the effects of the expansion on the indicators, it is observed that the creation of new campuses reduces the percentage of poverty in the municipalities, on average, by $1.5 \mathrm{pp}$. In addition, there is (weak) evidence that the policy increases the income per capita, the percentage of employed people with higher and medium levels. However, it should be noted that a longer period of time may be required for the effects of the expansion policy to have significant impacts on the economy.

Keywords: Impact evaluation, Expansion of Federal Universities, Municipalities indicators

- Recebido em: 2 de abril de 2019 - Aprovado em: 27 de fevereiro de 2020

DOI: http://dx.doi.org/10.1590/S1414-40772020000100009

Este é um artigo publicado em acesso aberto sob uma licença Creative Commons

https://creativecommons.org/licenses/by-nc/4.0/

Avaliação, Campinas; Sorocaba, SP, v. 25, n. 01, p. 155-177, mar. 2020 


\section{Introdução}

A educação se destaca como um fator estratégico no processo de crescimento e desenvolvimento econômico de regiões e países. Neste sentido, observa-se um destaque para as discussões sobre os impactos da educação superior no desenvolvimento econômico regional. Como destacam Rolim e Serra (2009), a Instituição de Ensino Superior (IES) tem um forte impacto no processo de desenvolvimento regional à medida que estabelecem vínculos e estão voltadas para a superação das questões da região em que estão.

Diante disso, espera-se que a universidade contribua para o desenvolvimento regional a partir da: ampliação da demanda gerada pelos recursos financeiros por meio dos salários dos professores e técnicos, pelos investimentos em obras, despesas de custeio e principalmente gastos dos alunos vindos de outra cidade; necessidade de modificação da infraestrutura local referente a habitação, transporte, lazer e serviços públicos demandados pelos agentes envolvidos com a universidade; contribuição para um aumento da produtividade, através da elevação do capital humano da população que impacta no processo produtivo (WILTGEN, 1991; SILVA, 2001).

Apesar do impacto econômico das universidades ser um tema com uma longa tradição na literatura sobre desenvolvimento regional, ele ainda é pouco estudado no Brasil. Mesmo no âmbito internacional, verifica-se uma predominância na literatura de estudos sob a metodologia de aplicação de multiplicadores de gastos e de matrizes insumo-produto. Já sob metodologia econométrica de efeito do tratamento sobre os tratados, seguindo uma técnica difundida na avaliação econômica de políticas públicas, foram encontrados os trabalhos de Vinhais (2013), Barbosa, Petterini e Ferreira (2014) e Niquito, Ribeiro e Portugal (2018), que estudaram o impacto da expansão das universidade federais no Brasil, e Neves (2015) que estudou o impacto da interiorização do Instituto Federal de Educação, Ciência e Tecnologia do Maranhão (IFMA).

É nessa perspectiva que este trabalho tem por objetivo estimar o impacto de curto prazo da expansão das Universidades Federais sobre indicadores econômicos, sociais e de mercado de trabalho dos municípios brasileiros. Além disso, são analisados quais fatores influenciaram os municípios a serem contemplados com um novo campi, campus ou uma Universidade Federal.

Para alcançar esses objetivos, optou-se por dividir o estudo em seis seções, incluindo esta introdução. A segunda seção versa sobre a política de expansão universitária no Brasil. A revisão da literatura sobre expansão universitária e seus efeitos são apresentados na terceira seção. A estratégia de identificação do efeito da política de interiorização das universidades 
federais, bem como a fonte e o tratamento dos dados e metodologia são discutidas na quarta seção. Em seguida, são analisados e discutidos os resultados e efeitos de curto prazo estimados da política. E, por fim, são tecidas as considerações finais.

\section{A política de expansão universitária no Brasil}

Desde 1998, o Brasil vem adotando uma série de medidas, buscando promover o crescimento do ensino superior público. Segundo Vinhais (2013), esse processo de expansão do ensino superior no Brasil pode ser dividido em três fases. Na primeira fase, entre 1998 e 2002, houve a expansão do número de vagas e cursos de nível superior nas sedes das universidades federais existentes.

$\mathrm{Na}$ fase posterior, de 2003 a 2006, ocorreu uma ampliação na oferta de cursos e vagas através da expansão em direção ao interior de diversos estados brasileiros, tendo em vista suprir a demanda das diversas regiões. Nesta fase, destaca-se a criação de novas universidades federais, bem como a criação e consolidação dos campi universitários das instituições já existentes.

Por fim, na terceira fase, foi instituído o Programa de Apoio a Planos de Reestruturação e Expansão das Universidades Federais (REUNI) por meio do Decreto no 6.096, de 24 de abril de 2007. O programa fazia parte de uma das ações integrantes do Plano de Desenvolvimento da Educação (PDE) acrescentar em reconhecimento ao papel estratégico das universidades federais para o desenvolvimento econômico e social.

Em sua formulação, o Reuni teve como principais objetivos: garantir às universidades as condições necessárias para a ampliação do acesso e permanência na educação superior; assegurar a qualidade por meio de inovações acadêmicas; promover a articulação entre os diferentes níveis de ensino, integrando a graduação, a pós-graduação, a educação básica e a educação profissional e tecnológica; e otimizar o aproveitamento dos recursos humanos e da infraestrutura das instituições federais de educação superior (BRASIL, 2009, p. 3).

De 2003 a 2010, houve um aumento de 45 para 59 universidades federais, representando uma ampliação de 31\%; e de 148 campi para 274 campi/unidades, representando um crescimento de 85\%. Ademais, entre 2003 e 2013, duas das regiões mais carentes de ensino superior - Norte e Nordeste - apresentaram expansão significativa da oferta. O percentual de crescimento das matrículas na região Nordeste, de 94\%, correspondeu ao dobro do registrado para o Sudeste e mais do triplo daquele registrado na região Sul. A região Norte teve a segunda maior taxa de crescimento (76\%) entre as regiões do país. Conforme a Secretaria de Educação 
Superior (Sesu), tais resultados são consequências dos investimentos da política de interiorização da universidade pública (BRASIL, 2015).

Nesse contexto, observe pela Figura 1 a expansão das Universidades Federais após 2002. Note que o maior aumento do número de campi ocorreu na região nordeste, seguido da região Sul. Além disso, também observa-se um aumento na região Norte do Brasil.

Figura 1- Expansão das Universidades Federais.

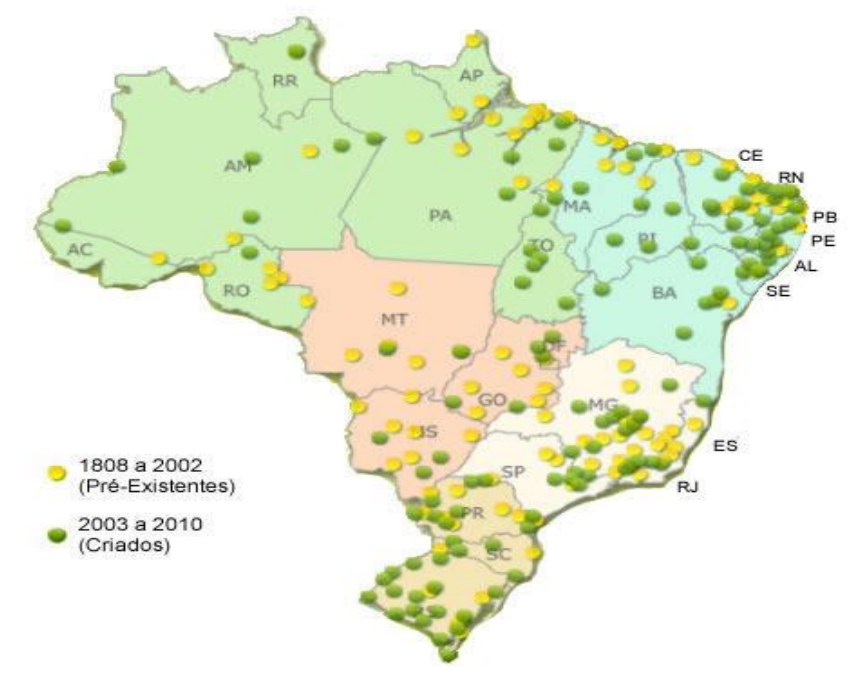

Fonte: BRASIL. Ministério da Educação. Expansão da educação superior e profissional e tecnológica: mais formação e oportunidades para os brasileiros. 2011. Disponível em: goo.gl/vPAq1G. Acesso em: 1 maio 2017.

As propostas de instalação de cursos, campi e universidades devem se basear em estudos preliminares das condições socioeconômicas das regiões, a fim de promover o desenvolvimento do país e redução das assimetrias regionais. Ao propor a instalação de novos campi e universidades, o Governo Federal estabeleceu critérios a serem analisados, de forma a estabelecer, objetivamente, prioridades e necessidades (BRASIL, 2015).

Dessa forma, segundo o Ministério da Educação (MEC), a expansão e interiorização da educação superior federal sucederam-se com base em três dimensões. Na dimensão social, destacou-se a priorização dos Territórios da Cidadania e aos munícipios populosos e com baixa renda per capita, além de municípios com elevado percentual de pobreza. Na dimensão geográfica, priorizaram-se os municípios com mais de 50.000 habitantes e microrregiões não atendidas, além de estados cuja oferta de educação superior estivesse abaixo da média nacional. 
Já na dimensão de desenvolvimento, buscou-se atender aos municípios com Arranjos Produtivos Locais identificados e aqueles situados no entorno de grandes investimentos (BRASIL, 2011)

Diante de todo panorama, espera-se que o objetivo principal da política de interiorização do ensino superior seja reduzir as desigualdades intra e inter-regionais, por meio do desenvolvimento socioeconômico gerado pela instituição.

\section{A literatura sobre os efeitos da expansão do ensino superior}

Uma universidade pode ter influências na economia regional no curto, médio e longo prazo. No curto prazo, observa-se impactos devido a gastos como: despesas e investimentos diretos da universidade; salários dos professores e funcionários universitários e respectivos impactos diretos sobre a demanda de bens e serviços; e os gastos dos estudantes (provenientes ou não da região). No médio prazo, com a consolidação da estrutura e funcionamento dos cursos, espera-se que tenha uma maior demanda por serviços. Enquanto que no longo prazo, há também efeitos de conhecimento, referentes basicamente ao lado da oferta, ou seja, referentes ao aumento do capital humano via nível de escolaridade e qualificação da mão de obra, maior investimento no setor de pesquisa, criação de novas empresas e atração de capital e mão-de-obra mais qualificada para a região (VINHAIS, 2013).

Segundo Schneider (2002), a universidade é considerada também um importante atrativo para a fundação de novos investimentos no município, por meio do fluxo de recursos financeiros injetados pela instituição, como pagamentos dos salários dos funcionários, professores, técnicos administrativos, concomitante à necessidade de obras, equipamentos e despesas de custeio e manutenção das instituições de ensino.

Neste sentido, de acordo com a argumentação de Florax (1992), é esperado que no curto prazo os efeitos gastos se sobreponham aos efeitos conhecimento, mas que tal relação se inverte no decorrer dos anos.

Bovo (2013) argumenta que diferente das demais atividades econômicas, as universidades públicas não possuem somente um impacto econômico estático (representado pelo efeito multiplicador do investimento), mas também apresentam impacto dinâmico sobre a economia em geral. E esse efeito dinâmico é exercido pela contribuição da universidade para aumentar o produto (local, regional e nacional) em função da sua atuação na formação e aperfeiçoamento do capital humano que, anualmente, se integra à produção social e à sua 
capacidade de transferir tecnologia para o sistema produtivo possibilitado pelo trabalho científico que ela desenvolve.

Ademais, no meio universitário são inseridos vários serviços (tais como livrarias, serviços de fotocópias, atividades de lazer, restaurantes, bares e infraestrutura de alojamento e transporte entre outros), desprendendo um processo de desenvolvimento e geração de empregos, impulsionando assim o setor de serviços e comércio (HOFF; MARTIN; SOPEÑA, 2011).

A interiorização das Universidades acaba provocando melhoras na infraestrutura escolar, hospitalar, cultural, de telecomunicação, lazer e transporte de muitos municípios, tornando-os atrativos para o estabelecimento da população (MORAES, 2000).

Dessa forma, o incentivo à educação, principalmente superior, leva ao local de implantação um crescimento acelerado. Esse processo rápido ocorre pela necessidade de o meio se adequar à nova realidade local, resultando no desenvolvimento por conta do aumento da demanda de docentes, técnicos e discentes no local. Ao mesmo tempo, os egressos do ensino médio sem opções de educação superior em sua região tendem a migrar, muitas vezes em caráter definitivo, para locais onde a oferta é mais ampla e diversificada. A região abandonada perde a oportunidade de fixar profissionais altamente qualificados e os estudantes sem condições financeiras de migrar para regiões mais propícias perdem a oportunidade de se qualificar (BRASIL, 2015).

No entanto, os estudos sobre os efeitos do ensino superior no Brasil são ainda incipientes. Muito disso decorre do fato do processo de expansão do Ensino Superior no Brasil ainda ser recente. Ademais, no Brasil, a literatura ainda é muito voltada ao desempenho das instituições no processo de inovação. Já a investigação da relação da IES e o desenvolvimento econômico, seja em termos locais ou regionais, ainda são escassos.

Destacam-se na literatura os estudos internacionais que buscam medir o impacto econômico de novas universidades a partir da aplicação de multiplicadores de gastos e do uso de matrizes insumo-produto, concentrando-se nos efeitos locais de curto prazo. Turner (1997), por exemplo, aduz que, por meio da aplicação de multiplicador, cada nova unidade monetária introduzida na economia local gera novas atividades e produz novos empregos.

Estudos desenvolvidos para análise do impacto econômico da Universidade de Aberdeen na economia do Nort Eeast of Scotland na óptica da despesa (BATTU; FINCH, 1998) mostram que a maior componente da despesa da universidade é a relacionada com o custo dos funcionários. Além do emprego direto de 2375 funcionários, através do multiplicador de 
emprego de 1,61 a universidade gerou um aumento de empregos locais avaliado em 1449, em que o setor de serviços detém a maioria. Para o Brasil, Kuresk e Rolim (2009) estimaram um multiplicador de renda de $\mathrm{R} \$ 1,94$ para cada real pago em salário nas universidades federais brasileiras.

Caldarelli, Camara e Perdigão (2015) analisou a relação entre as IES e o desenvolvimento econômico no Paraná, utilizando a metodologia de dados em painel e indicadores agregados em três dimensões: i) emprego/renda; ii) educação; e iii) saúde. O resultado mostrou que os municípios que possuíam IES estaduais apresentaram indicadores de desenvolvimento humano maiores que aqueles que não tinham, com maior impacto para emprego/renda, enquanto para educação e saúde esses indicadores ainda eram modestos.

Siegfried, Sanderson e Mchenry (2007) criticam essas abordagens, aplicação de multiplicadores de gastos e matrizes insumo-produto, utilizadas nos estudos sobre o impacto econômicos das universidades por não apresentarem natureza contrafactual, ou seja, por não serem capazes de comparar o desempenho da presença da universidade ante o desempenho caso esta não existisse. Ademais, criticam também a incapacidade dessas metodologias de identificar o real impacto causado pela universidade em detrimento dos efeitos causados por outras razões.

Na literatura nacional, os trabalhos de Vinhais (2013), Barbosa, Petterini e Ferreira (2014), Neves (2015) e Niquito, Ribeiro e Portugal (2018) utilizam uma metodologia para captar o efeito do tratamento sobre os tratados baseada em análise contrafactual para estimar os impactos da presença de novas universidades/institutos sobre o desenvolvimento local.

Vinhais (2013) analisou os efeitos de curto prazo da expansão da rede de universidades federais no Brasil e observou que o efeito médio do tratamento sobre os tratados foi de 3,3\% sobre a renda domiciliar per capita municipal e que os municípios menores apresentaram os maiores efeitos de tratamento.

De acordo com os resultados obtidos por Barbosa, Petterini e Ferreira (2014), em municípios menores, o efeito de curto prazo do surgimento de campi de universidades federais nos municípios brasileiros foi significante, mas não ocorreram efeitos consideráveis de longo prazo. Já em municípios maiores ocorreu o inverso, no curto prazo os efeitos foram insignificantes, enquanto no longo prazo os novos campi promoveram ganhos para as economias locais.

Para investigar os efeitos da criação das novas universidades federais sobre o desenvolvimento das economias locais, Niquito, Ribeiro e Portugal (2018) estimam um modelo 
de diferenças em diferenças, para capturar os efeitos de transbordamento que a criação de novos campi em um determinado município possa ter tido sobre os municípios vizinhos. Os autores observam que melhora na renda domiciliar per capita das famílias e redução da taxa de fecundidade e, além disso, eles observam que municípios de menor porte tendem a ser mais afetados pela criação de um novo campus.

A interiorização e expansão do IFMA é avaliada por Neves (2015), que observou o impacto positivo da sobre desempenho escolar do Ensino Fundamental nos municípios contemplados com a implantação. Mais especificamente, o efeito foi positivo nas notas do IDEB.

Com esteio nessa discussão, essa pesquisa tende a contribuir com a literatura dois aspectos, a saber: i) estimar a chance dos municípios em receber um novo campus (ou campi); e, ii) estimar os efeitos de curto prazo da expansão das Universidades Federais sobre indicadores econômicos, sociais e de mercado de trabalho.

\section{Estratégia de identificação}

Com intuito de estimar o impacto da expansão das Universidades Federais sobre as economias municipais consideram-se como unidade de observação os municípios brasileiros em dois períodos de tempo (pré-tratamento e pós-tratamento), excluindo-se os 114 municípios que já possuíam campus antes de 2003, como se observa na Figura 1 - pré-existentes, 1808 a 2002.

Os municípios tratados são os que receberam algum campus na década anterior, após o ano de 2002. Esses municípios são identificados a partir de pesquisa sobre o histórico das Universidades Federais, para identificar as expansões e criações de novos campi por essas instituições. Sendo assim, o estudo almeja inferir sobre o efeito médio do tratamento sobre os tratados (Average Treatment Effect on the Treated - ATT), de modo que o ATT = E[Y(1) - Y(0) | $D=1]$, ou seja, busca avaliar os efeitos sobre os municípios beneficiados com a expansão universitária.

Nesse sentido, questiona-se o que ocorreria com alguns indicadores econômicos, sociais e do mercado de trabalho dos municípios beneficiados com o campus, caso ele não tivesse sido implementado. Visto que é impossível observar simultaneamente informações de um mesmo município recebendo o campus e não recebendo, se faz necessário selecionar um grupo de controle para responder pelo contrafactual. 
Rosenbaum e Rubin (1983) propuseram a metodologia de Pareamento por Escore de Propensão (PSM), que busca construir um grupo de controle consistente e o mais similar possível ao grupo de tratamento em termos de determinadas características observáveis, partindo da concepção de que quanto mais parecidos são os grupos, menor será o viés.

Desse modo, cada município do grupo de tratamento teria pelo menos um par no grupo de controle, representando, assim, o resultado que ele obteria caso não fosse tratado. A diferença nos resultados desses municípios seria dada apenas pelo tratamento.

$\mathrm{O}$ escore de propensão nada mais é do que a probabilidade condicional de receber o tratamento, dada às variáveis observadas $\mathrm{X}$ antes do tratamento. Assim, o escore de propensão é definido como:

$$
P(X)=\operatorname{Pr}[T=1 \mid X]
$$

A estimação do escore de propensão é geralmente feita por meio de procedimentos paramétricos para estimação de probabilidade, como os modelos logit ou probit (MENEZES FILHO, 2012). No caso do modelo logit, o escore de propensão é dado por:

$$
\hat{p}(x)=\frac{\exp (x \widehat{\beta})}{1+\exp (x \widehat{\beta})}
$$

Conforme Khandker, Koolwal e Samad (2010) para que o efeito médio do tratamento sobre o tratado (ATT) seja identificado e estimado consistentemente, duas hipóteses centrais precisam ser atendidas para validar o Pareamento por Escore de Propensão:

(i) Seleção nos observáveis, de modo que os resultados potenciais são independentes do tratamento condicionado nas características observáveis; e

(ii) Existe suporte comum, de forma que não existem observações em que o pesquisador saiba se a unidade foi ou não submetida ao tratamento ao observar somente as covariadas. Assim, requer que haja sobreposição nos escores de propensão entre as unidades dos grupos de tratado e controle. Desse modo, é necessário que:

$$
0<\operatorname{Pr}[D=1 \mid X]<1
$$

Então, baseado no escore de propensão, selecionam-se o grupo de comparação, ou seja, pareamos unidades tratadas e não tratadas. Existem diferentes metodologias de seleção do grupo de comparação. Segundo Becker e Ichino (2002), as metodologias mais utilizadas na literatura de avaliação são Pareamento Estratificado (Stratification Matching - SM), Pareamento por 
Vizinho mais Próximo (Nearest Neighbor Matching - NNM), Pareamento por Raio (Radius Matching - RM), Pareamento de Kernel (Kernel Matching - KM).

No presente estudo foi utilizado o Pareamento por NNM, em que para cada unidade tratada é procurada uma unidade não tratada com o escore de propensão mais próximo, ou seja, o pareamento é realizado de forma a minimizar a diferença absoluta entre o escore de propensão da unidade tratada e não tratada.

Ademais, para verificar a adequabilidade do grupo de controle construído após o pareamento, o presente trabalho se baseará na medida de equilíbrio definida por Cochran e Rubin (1973), sendo que as unidades de controle selecionadas constituem um contrafactual adequado se a diferença padronizada - (média da variável no grupo de tratamento - média da variável no grupo de controle) /desvio-padrão do grupo de controle - não exceder o valor de 0,25 desvio padrão.

Segundo Menezes Filho (2012), uma das críticas aos métodos baseados nas duas hipóteses acima é que, ao tomar a decisão de participar ou não, os municípios olham não só para um vetor de características observáveis, mas também para características que não são observadas pelo avaliador. Se estas características não observáveis forem correlacionadas tanto com a decisão de participar do programa como com o resultado potencial deste município, o método do pareamento não conseguirá eliminar o viés de seleção, e a estimação estará sujeita a um viés. Dessa forma, para mitigar o viés de seleção, foi combinado o pareamento com o método de diferenças em diferenças.

Uma vez estimado o escore de propensão e feito o pareamento, estima-se o modelo de diferenças em diferenças, que tem a vantagem de controlar para características não observáveis dos municípios que sejam invariantes no tempo. $\mathrm{O}$ estimador de diferenças em diferenças calcula uma dupla diferença de médias, a diferença entre as médias da variável de impacto para os grupos de tratamento e controle antes e depois do programa, e a diferença entre essas diferenças.

Considerando um painel de 2 períodos, pode-se obter uma estimativa do ATT por meio do parâmetro $\beta$, conforme Foguel (2012):

$$
Y_{i t}=\delta+X_{i t}^{\prime} \alpha+\gamma T_{i t}+\rho t_{i t}+\beta\left(T_{i t} t_{i t}\right)+\varepsilon_{i t}
$$

Em que: $Y_{i t}$ é o indicador de impacto avaliado no município i no período $\mathrm{t}, t_{i t}$ é uma variável binária que assume valor zero para o período pré-tratamento e valor um para o período 
pós-tratamento; $X_{i t}^{\prime}$ é um vetor de covariadas; $T_{i}$ expressa tratamento ou controle $\left(T_{i}=1 \mathrm{em}\right.$ ambos os períodos se o município foi tratado e $T_{i}=0$ se não foi tratado); $\varepsilon_{i t}$ é um termo de erro; $\delta, \alpha, \gamma, \rho$ são parâmetros e $\beta$ é o parâmetro que indica o efeito causal da política/programa.

No estudo, os indicadores de impacto $\left(Y_{i t}\right)$ utilizados para estimar o efeito de tratamento sobre os tratados (ATT) contemplam a área econômica (renda per capita), social (índice de Gini, percentual de pobres) e de mercado de trabalho (ocupados nos setores de serviços, indústria, comércio; percentual de ocupados com ensino médio e ensino superior). Os quais foram extraídos do Atlas Brasil, considerando os anos de 2000 e 2010 por ser antes e após a política de expansão universitária.

De acordo com a literatura espera-se que a criação de novos campi possa contribuir com o crescimento e desenvolvimento dos municípios beneficiados. Dessa forma, espera-se que haja um efeito positivo sobre a renda per capita, no mercado de trabalho e um efeito negativo no percentual de pobres e índice de Gini. No entanto, vale ressaltar que talvez seja necessário maior período de tempo para que se observem os efeitos da política de expansão universitária. Em outros termos, pode-se dizer que mudança no mercado de trabalho seja, por exemplo, um indicador de médio ou longo prazo, enquanto a renda possa ter efeito já no curto prazo.

Para mitigar viés por omissão de variáveis e também para captar o efeito líquido do tratamento, utilizam-se características observáveis dos municípios como covariadas no vetor X, a saber: Percentual de pessoas de 18 anos ou mais com fundamental completo, Percentual de pessoas de 18 anos ou mais com médio completo, Percentual de pessoas de 25 anos ou mais com superior completo, Percentual da população em domicílios com água encanada, Percentual da população em domicílios com coleta de lixo, Percentual da população em domicílios com energia elétrica, Taxa de atividade - 18 anos ou mais, Taxa de envelhecimento e População urbana. As variáveis são extraídas do Atlas Brasil referente aos anos de 2000 e 2010.

Espera-se que o grupo de controle represente um contrafactual adequado para o grupo de tratamento, neste sentido o método DD requer algumas hipóteses:

(i) Não ocorra qualquer mudança após o início da intervenção (implantação de campi/campus) capaz de afetar de forma heterogênea os grupos de tratamento e controle; e,

(ii) A trajetória temporal da variável resultado tem de ser similar para o grupo de tratamento e de controle, de forma que ambos os grupos reagiriam de forma semelhante aos demais fatores que impactam na variável de resultado antes da intervenção (FOGUEL, 2012). 
Assim, se as hipóteses forem satisfeitas, a diferença nos indicadores de resultados entre os grupos (tratado e controle) podem ser atribuídas ao efeito causal do tratamento (isto é, ter sido contemplado com um novo campus ou campi). Além disso, ao combinar o método de PSM com o modelo de diferenças em diferenças, espera-se que o efeito do tratamento estimado não seja viesado, uma vez que o PSM minimiza o viés proveniente da distribuição de atributos observáveis e de ausência de suporte comum, enquanto que o método DD reduz o viés de seleção (PEIXOTO; ANDRADE; AZEVEDO, 2008).

Além disso, para tentar captar impactos de curto e médio prazo, utilizam-se duas especificações de acordo com o ano de criação do campus, sendo:

E1: Em que $T_{(1) i}=1$ para caso o município tenha recebido o campus independente do ano em que ocorreu, mostrando, assim, o impacto da política independente do ano de implantação do campus (2003-2010);

E2: Em que $T_{(2) i}=1$ para caso o município tenha recebido o campus após o ano de 2006, mostrando, assim, o impacto da política sobre os municípios que receberam o campus mais recente (2007-2010).

Deste modo, o modelo com a especificação 2 seria:

$$
Y_{i t}=\delta+X_{i t}^{\prime} \alpha+\gamma T_{(1) i t}+\varphi T_{(2) i}+\rho t_{i t}+\beta_{1}\left(T_{(1) i t} t_{i t}\right)+\beta_{2}\left(T_{(2) i t} t_{i t}\right)+\varepsilon_{i t}
$$

Assim, conforme Barbosa, Petterini e Ferreira (2014), o efeito da política de expansão para os municípios tratados seria $\beta_{1}$ e para os municípios tratados mais recentemente seria $\beta_{1}+$ $\beta_{2}$.

\section{Análise e discussão dos resultados}

\subsection{Pareamento dos grupos de tratado e controle}

Primeiramente, com o intuito de verificar quais variáveis tiveram relação com a implantação de algum campus da Universidade Federal na década de 2000, foi estimado um modelo logit para um período pré-tratamento. Para estimar essa probabilidade são consideradas informações que contribuem com a implantação de Universidades Federais nos municípios brasileiros, além das consultas as pesquisas relacionadas ao tema como, por exemplo, Barbosa, Petterini e Ferreira (2014), Vinhais (2013) e Niquito, Ribeiro e Portugal (2018). 
No referido modelo, a variável dependente é a indicadora do tratamento (receber algum campus de universidade federal entre 2003-2010), enquanto as características que afetam a chance do município ser beneficiado com a política de expansão universitária é composto pelas variáveis reportadas no Quadro 1.

Quadro 1- Descrição das variáveis utilizadas no pareamento, ano e fonte

\begin{tabular}{|l|c|l|}
\hline \multicolumn{1}{|c|}{ Variável } & Ano & \multicolumn{1}{|c|}{ Fonte } \\
\hline População do município & 2000 & Censo Demográfico do IBGE \\
\hline $\begin{array}{l}\text { Dummy indicando se o município tinha população superior a 50 mil } \\
\text { habitantes }\end{array}$ & 2000 & Censo Demográfico do IBGE \\
\hline $\begin{array}{l}\text { Dummy indicando se o município está em microrregião que já possuía } \\
\text { um campus de alguma instituição federal }\end{array}$ & 2002 & $\begin{array}{l}\text { Censo da Educação Superior do } \\
\text { INEP/MEC }\end{array}$ \\
\hline Dummy indicando se o município já tem campus particular & 2002 & $\begin{array}{l}\text { Censo da Educação Superior do } \\
\text { INEP/MEC }\end{array}$ \\
\hline Dummy indicando se o município pertence à região metropolitana & 2000 & IBGE \\
\hline $\begin{array}{l}\text { Dummy indicando se o município apresenta receita corrente per capita } \\
\text { de até R\$ 1 mil }\end{array}$ & 2003 & $\begin{array}{l}\text { Dados Contábeis } \\
\text { Municípios do FINBRA - STN }\end{array}$ \\
\hline $\begin{array}{l}\text { Dummies indicando se o PIB do município era o primeiro, segundo ou } \\
\text { terceiro maior dentre os municípios da microrregião. }\end{array}$ & 2002 & $\begin{array}{l}\text { Bases de Dados das Contas } \\
\text { Regionais do IBGE }\end{array}$ \\
\hline Índice de Desenvolvimento Humano & 2000 & IPEA \\
\hline
\end{tabular}

Fonte: Elaborado pelos autores.

Note pela Tabela 1 que a existência de campus particular, Dummies indicando se o PIB do município era o primeiro, segundo ou terceiro maior dentre os municípios da microrregião e se o município tinha população superior a 50 mil habitantes aumentam a probabilidade de um município receber um campus da Universidade Federal, enquanto $\ln$ (da população) teve efeito negativo, porém o coeficiente é próximo de zero.

A partir dos resultados expostos na Tabela 1, realiza-se o pareamento com base no escore de propensão, que corresponde à probabilidade condicional de receber um campus de universidade federal entre 2003-2010 dada às variáveis observadas antes do tratamento. Dessa forma, baseado no escore de propensão, foi selecionado o grupo de controle, utilizando o Pareamento por $N N M$ com 2 vizinhos, em que para cada município tratado são escolhidos dois municípios não tratados com o escore de propensão mais próximos. Ressalta-se que o mesmo município poderia ser selecionado como controle para mais de uma unidade municipal tratada. 
Tabela 1 - Resultados estimados para captar a Probabilidade de Instalação de um campus de IES Federal

\begin{tabular}{c|c|c}
\hline \multicolumn{2}{c}{ Variável dependente: Pr (receber algum campus de Universidade Federal entre 2003-2010) } \\
\hline Variáveis explicativas & Coeficientes & Erro padrão \\
\hline Região metropolitana & 0,296 & 0,2634 \\
IDHM & $-1,706$ & 1,324 \\
Ln população & $-1,419 \mathrm{E}-07 * * *$ & $4,276 \mathrm{E}-08$ \\
Tem campus particular & $0,640^{*}$ & 0,2867 \\
Microrregião tem campus $^{\text {PIB microrregião 1 }}$ & 0,412 & 0,3186 \\
PIB microrregião 2 & $3,591^{*} * *$ & 0,4461 \\
PIB microrregião 3 & $2,700^{*} * *$ & 0,4458 \\
Receita per capita baixa & $1,595^{*} *$ & 0,5454 \\
População >50 mil & $-0,029$ & 0,2935 \\
Constante & $0,802^{* *}$ & 0,2528 \\
\hline
\end{tabular}

Fonte: Elaborado pelos autores.

Nota: $* * * \mathrm{p}<0.01, * * \mathrm{p}<0.05, * \mathrm{p}<0.1$

A Figura 2 corresponde à distribuição do escore de propensão e, a ausência de casos na estratificação superior indica que não havia unidades de tratamento incomparáveis. As estratificações do meio mostram a proximidade entre as unidades de tratamento e as unidades de controle correspondentes. Enquanto a estratificação final mostra as unidades de controle incomparáveis, que não serão utilizadas.

Figura 2 - Distribuição dos escores de propensão dos grupos de tratamento e controle, com e sem o pareamento

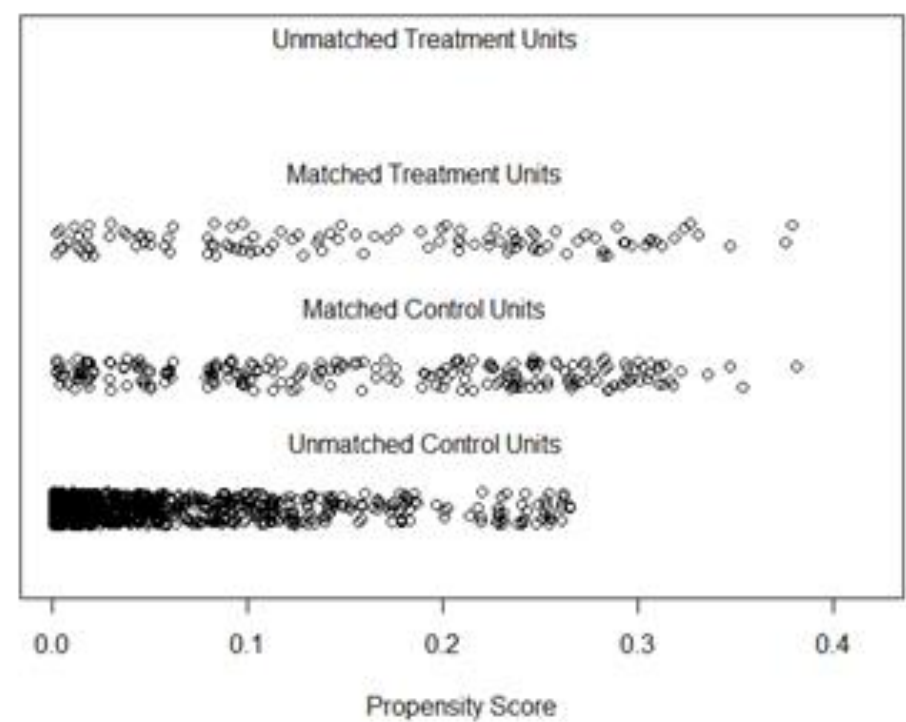

Fonte: Elaborado pelos autores. 
Analisando os escores de propensão, Figura 3, observa-se que os histogramas antes do pareamento (à esquerda) se diferem bastante, enquanto que os histogramas após o pareamento (à direita) estão mais homogêneos, indicando que o pareamento foi consistente. Ademais, para verificar a adequabilidade do grupo de controle construído após o pareamento, são calculadas as diferenças padronizadas das médias para verificar o grau de equilíbrio entre os grupos para as variáveis relevantes antes da ocorrência do tratamento.

\section{Figura 3 - Histogramas dos escores de propensão dos grupos de tratamento e controle, antes e depois do pareamento}
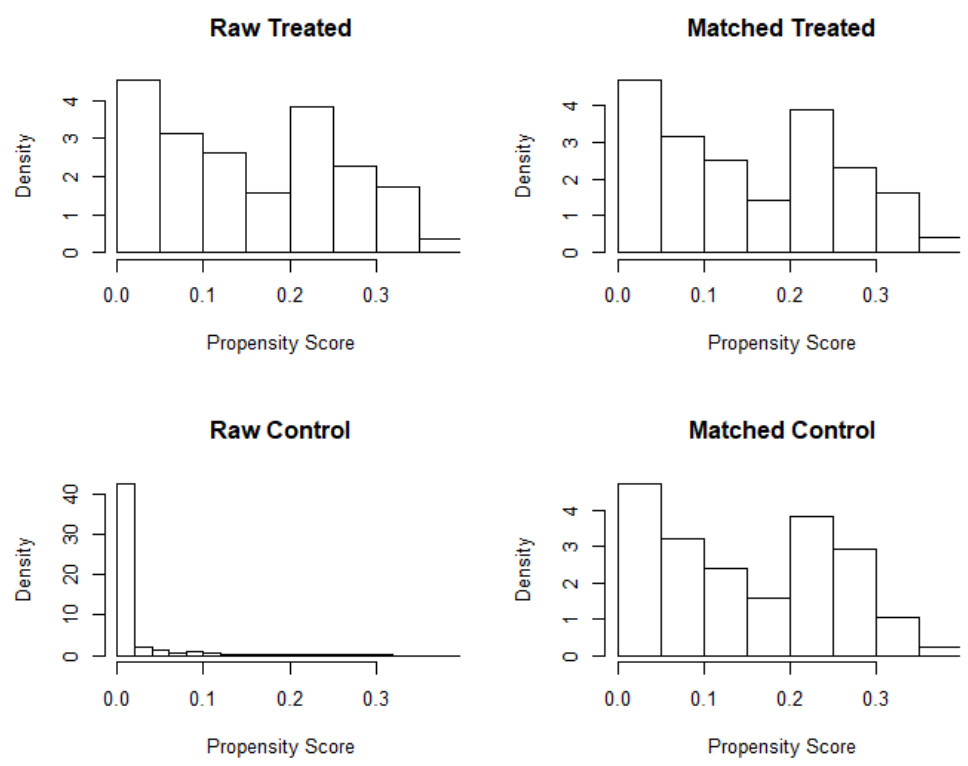

Fonte: Elaborada pelos autores.

Observe pela Figura 4 que o método de pareamento resultou em uma redução significativa das diferenças padronizadas entre os grupos de tratamento e de controle. Note as diferenças padronizadas entre os grupos de tratamento e controle pareado (bola cinza) e entre os grupos de tratamento e controle não pareado (bola laranja) - que consideram em sua composição todos os municípios não tratados da base de dados. 


\section{Figura 4 - Gráfico de Equilíbrio das Diferenças padronizadas entre os grupos de tratamento e controle após o pareamento}

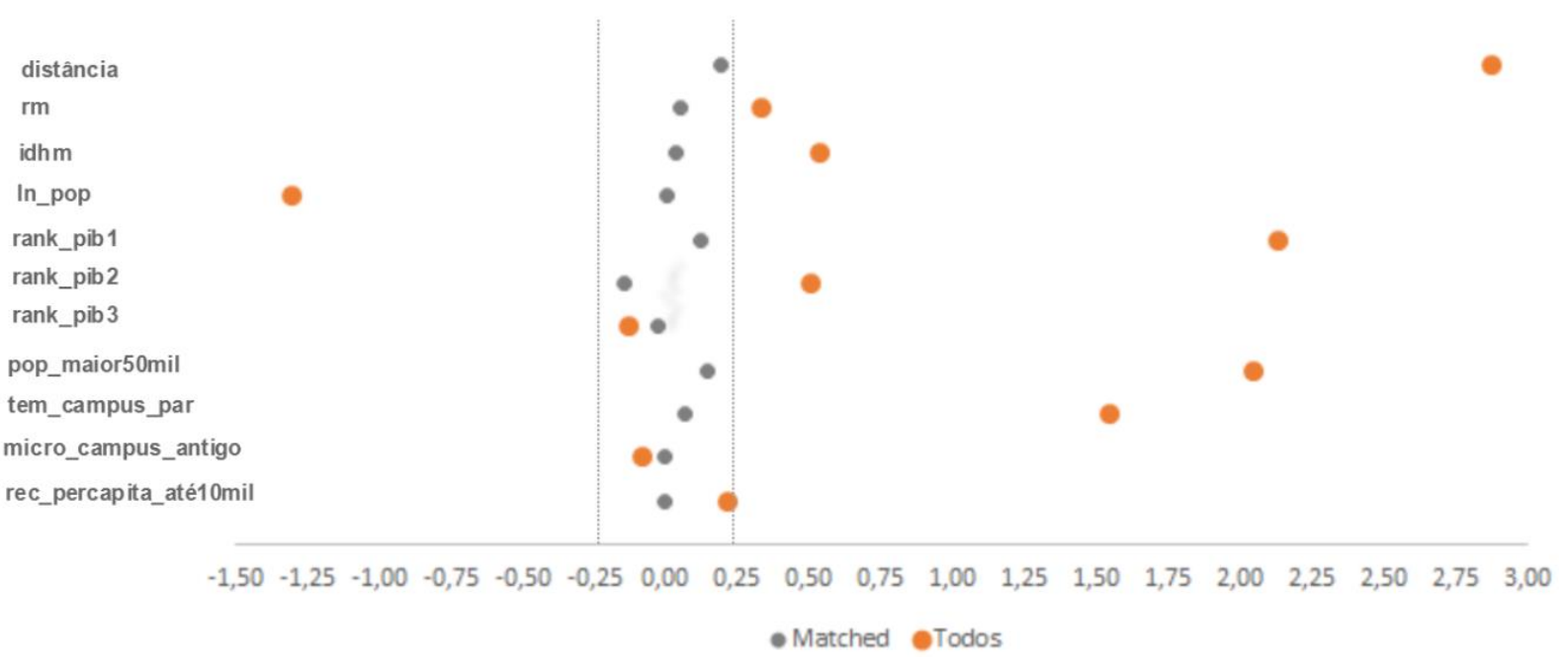

Fonte: Elaborado pelos autores.

Adicionalmente, a Figura 4, Gráfico de equilíbrio, evidencia que as unidades de controle selecionadas constituem um contrafactual adequado, visto que as diferenças padronizadas não excedem o valor de 0,25 desvio padrão, enquanto não é verificado no grupo de não pareados, segundo o critério de Cochran e Rubin (1973). Sendo assim, o grupo de controle selecionado por meio da técnica de pareamento a partir do escore de propensão tende a representar os municípios que receberam algum campus com a política de expansão universitária caso não tivessem recebido.

\subsection{Análises dos efeitos da expansão universitária}

Esta seção contempla a análise e discussão dos resultados da pesquisa que versa sobre a mensuração do efeito da política de expansão das Universidades Federais sobre as economias municipais considerando indicadores econômicos, sociais e do mercado de trabalho. Vale ressaltar que os resultados são aferidos a partir do pareamento pelo escore de propensão, seguindo a estratégia de adotar duas especificações de acordo com o ano de criação do campus com intuito de observar impactos de curto e médio prazo.

$\mathrm{Na}$ especificação E1, o coeficiente da variável binária Ano*Tratado capta o efeito de tratamento sobre os municípios que receberam campus, independente do ano de implantação do campus (2003-2010). Enquanto a especificação E2, o efeito da política pode ser observado por 
Ano*Tratapós2006 quando somado com o da variável Ano*Tratado mostra o efeito de tratamento sobre os municípios tratados após 2006. Como teste de robustez optou-se por estimar os mesmos modelos com as mesmas especificações considerando o estimador de efeitos fixos.

A partir dos resultados expostos na Tabela 3, observa-se que utilizando o método de Efeito Fixo, a criação de novos campi teve efeito sobre a renda per capita, percentual de ocupados com nível superior, percentual de ocupados com nível médio e percentual de pobres. Já sobre as demais variáveis não foi possível verificar relação de impacto.

Analisando o impacto na renda per capita verifica-se um efeito positivo de 3,4\%. Já quando se parte do efeito tratamento após 2006, não se verificou impacto. Isso pode ter decorrido do fato de ser necessária uma maturação temporal maior para que a política gere efeitos sobre a renda per capita do município. O resultado encontrado vai ao encontro com o estudo de Vinhais (2013) que estimou um efeito médio do tratamento sobre os tratados de 3,3\% sobre a renda domiciliar per capita e de Barbosa, Petterini e Ferreira (2014) que encontrou resultado similar de que a implantação dos novos campi teve impacto de $4,4 \%$ sobre a renda per capita (pareamento 1). Dessa forma, nos estudos encontrados percebe-se que a expansão das Universidades trouxe impacto positivo da renda per capita dos municípios beneficiados.

Esse resultado pode ser em decorrência da ampliação da demanda gerada pelos recursos financeiros por meio dos salários dos professores e técnicos, pelos investimentos em obras, despesas de custeio e de gastos dos alunos vindos de outra cidade. Além disso, são inseridos vários serviços, tais como livrarias, serviços de fotocópias, atividades de lazer, restaurantes, bares e infraestrutura de alojamento e transporte entre outros, desprendendo um processo de desenvolvimento e geração de empregos, impulsionando assim a economia do município.

Note também que a política gerou resultado positivo de 0,58 p.p. sobre o percentual de ocupados com nível superior e não houve efeito para os tratados após 2006. Esse fato corrobora com a ideia de que é necessário certo período de tempo para que os alunos concluam a graduação e, posteriormente, entrem no mercado de trabalho, caso o município absorva a mão-de-obra qualificada. Observam-se também efeitos de conhecimento, referentes à expansão do capital humano (aumento de escolaridade, qualificação da mão-de-obra) e possível crescimento da área de pesquisa, que pode impactar no processo produtivo dos municípios.

Sobre o percentual de ocupados com nível médio, os resultados mostram que houve um impacto positivo de 1,1 p.p. sobre os municípios com campi inaugurados até 2006 , o que pode ser em função da abertura de vagas nas próprias Universidades para servidores técnicos 
administrativos e/ou prestadores de serviços terceirizados. Por outro lado, observa-se impacto negativo de -0,83 p.p. sobre os municípios beneficiados após 2006 , que pode ter ocorrido pelo acesso ao ensino superior das pessoas com ensino médio completo, o que pode influenciar na decisão de se dedicar apenas aos estudos e, por conseguinte, se retirar do mercado de trabalho para cursar a Universidade.

De forma geral, não foi possível verificar impactos de curto prazo, visto que os resultados dos modelos de especificação E2 não foram estatisticamente significantes. Note que, pelo estimador de Efeito Fixo, observa-se que a criação de campi gera, em média, uma redução de 1,27 p.p. sobre o percentual de pobres nos municípios. Enquanto que pelo método de Diferenças em Diferenças, a redução no percentual de pobres é de 1,5 p.p. Sendo assim, pode-se inferir que, ao menos no curto prazo, a política de expansão universitária tende a contribuir para a redução da pobreza.

Em síntese, pode-se inferir que a criação de novos campi impacta negativamente 1,5 p.p. no percentual de pobres dos municípios, em média. Além disso, observa-se evidência (fraca) de efeitos positivos na renda per capita, no percentual de ocupados com nível superior e com nível médio e nos demais indicadores não foram identificados efeitos.

Contudo, vale ressaltar que talvez seja necessária uma defasagem temporal maior para que os efeitos da política de expansão causem impactos significativos sobre a economia em determinados indicadores. Isso decorre porque para a Universidade gerar forte impacto no desenvolvimento local, é necessário que os municípios se adequem à nova realidade e consigam absorver os benefícios que a política de expansão das Universidades pode demandar, assim, certo tempo de maturação. 
Tabela 3: Os efeitos de curto prazo estimados da política de expansão das Universidades Federais.

\begin{tabular}{|c|c|c|c|c|c|c|c|c|c|c|c|c|c|c|c|c|c|}
\hline \multirow{3}{*}{ Estimador } & \multirow{3}{*}{$\begin{array}{c}\text { Indicadores } \\
\text { Modelos }\end{array}$} & \multirow{2}{*}{\multicolumn{2}{|c|}{$\begin{array}{c}\text { Econômicos } \\
\begin{array}{c}\text { Ln (renda per } \\
\text { capita) }\end{array}\end{array}$}} & \multicolumn{10}{|c|}{ Mercado de Trabalho } & \multicolumn{4}{|c|}{ Sociais } \\
\hline & & & & \multicolumn{2}{|c|}{$\begin{array}{l}\text { Ocupados no setor } \\
\text { de serviços }\end{array}$} & \multicolumn{2}{|c|}{$\begin{array}{c}\text { Ocupados no } \\
\text { setor do comércio }\end{array}$} & \multicolumn{2}{|c|}{$\begin{array}{l}\text { Ocupados no setor } \\
\text { da indústria }\end{array}$} & \multicolumn{2}{|c|}{$\begin{array}{l}\text { Ocupados com } \\
\text { nível superior }\end{array}$} & \multicolumn{2}{|c|}{$\begin{array}{l}\text { Ocupados com } \\
\text { nível médio }\end{array}$} & \multicolumn{2}{|c|}{$\begin{array}{l}\text { Percentual de } \\
\text { pobres }\end{array}$} & \multicolumn{2}{|c|}{ Gini } \\
\hline & & E1 & E2 & E1 & E2 & E1 & E2 & E1 & E2 & E1 & E2 & E1 & E2 & E1 & E2 & E1 & E2 \\
\hline \multirow{14}{*}{ Efeito fixo } & Ano*Tratado & $0.034 * *$ & 0.027 & -0.119 & -0.240 & 0.0344 & -0.009 & 0.033 & 0.333 & $0.579 * *$ & $0.549^{*}$ & 0.250 & $1.097^{* *}$ & $1.268 * *$ & $-1.445^{*}$ & 0.005 & 0.006 \\
\hline & & $(0.016)$ & $(0.019)$ & $(0.416)$ & $(0.513)$ & $(0.265)$ & $(0.327)$ & $(0.415)$ & $(0.512)$ & $(0.238)$ & $(0.294)$ & $(0.433)$ & $(0.529)$ & $(0.621)$ & $(0.770)$ & $(0.005)$ & $(0.006)$ \\
\hline & Ano*Tratapós2006 & & $\begin{array}{c}0.0152 \\
(0.0257)\end{array}$ & & $\begin{array}{c}0.276 \\
(0.683)\end{array}$ & & $\begin{array}{l}0.0984 \\
(0.435)\end{array}$ & & $\begin{array}{l}-0.681 \\
(0.682)\end{array}$ & & $\begin{array}{l}0.0671 \\
(0.390)\end{array}$ & & $\begin{array}{c}-1.926 \text { **** } \\
(0.704)\end{array}$ & & $\begin{array}{c}0.398 \\
(1.023)\end{array}$ & & $\begin{array}{l}-0.003 \\
(0.008)\end{array}$ \\
\hline & $\mathrm{R}^{2}$ & 0.893 & 0.894 & 0.328 & 0.329 & 0.414 & 0.414 & 0.143 & 0.145 & 0.843 & 0.843 & 0.940 & 0.942 & 0.904 & 0.905 & 0.570 & 0.570 \\
\hline & perc_18oumais_fund_comp & Sim & Sim & Sim & Sim & Sim & Sim & $\operatorname{Sim}$ & Sim & & & & & & & & \\
\hline & perc_med_comp_18 & Sim & Sim & Sim & Sim & Sim & Sim & Sim & Sim & & & & & & & & \\
\hline & perc_sup_comp_25 & Sim & Sim & Sim & Sim & Sim & Sim & Sim & Sim & & & & & & & & \\
\hline & Água & Sim & Sim & Sim & Sim & Sim & Sim & Sim & Sim & Sim & Sim & Sim & Sim & Sim & Sim & Sim & Sim \\
\hline & Lixo & Sim & Sim & Sim & Sim & Sim & Sim & Sim & Sim & Sim & Sim & Sim & Sim & Sim & Sim & Sim & Sim \\
\hline & Luz & Sim & Sim & Sim & Sim & Sim & Sim & Sim & Sim & Sim & Sim & Sim & Sim & Sim & Sim & Sim & Sim \\
\hline & tx_atividade_18oumais & Sim & Sim & Sim & Sim & Sim & Sim & Sim & Sim & & & & & Sim & Sim & Sim & Sim \\
\hline & Urbano & Sim & Sim & Sim & Sim & Sim & Sim & Sim & Sim & Sim & Sim & Sim & Sim & Sim & Sim & Sim & Sim \\
\hline & tx_analf_18oumais & & & & & & & & & & & & & Sim & Sim & Sim & Sim \\
\hline & tx_envelhecimento & & & & & & & & & & & & & Sim & Sim & Sim & Sim \\
\hline \multirow{13}{*}{$\begin{array}{l}\text { Diferenças } \\
\text { em } \\
\text { diferenças }\end{array}$} & Ano*Tratado & $\begin{array}{c}0.006 \\
(0.025)\end{array}$ & $\begin{array}{l}-0.000 \\
(0.033)\end{array}$ & $\begin{array}{c}-0.0675 \\
(0.770)\end{array}$ & $\begin{array}{l}-0.508 \\
(0.896)\end{array}$ & $\begin{array}{c}0.113 \\
(0.545)\end{array}$ & $\begin{array}{c}0.006 \\
(0.759)\end{array}$ & $\begin{array}{c}-0.0663 \\
(1.018)\end{array}$ & $\begin{array}{c}0.249 \\
(1.088)\end{array}$ & $\begin{array}{c}0.459 \\
(0.505)\end{array}$ & $\begin{array}{c}0.208 \\
(0.518)\end{array}$ & $\begin{array}{c}0.450 \\
(0.998)\end{array}$ & $\begin{array}{c}1.014 \\
(1.169)\end{array}$ & $\begin{array}{c}-1.539^{*} \\
(0.892)\end{array}$ & $\begin{array}{l}-1.672 \\
(1.190)\end{array}$ & $\begin{array}{c}0.003 \\
(0.007)\end{array}$ & $\begin{array}{c}0.006 \\
(0.008)\end{array}$ \\
\hline & Ano*Tratapós2006 & & $\begin{array}{c}0.017 \\
(0.039)\end{array}$ & & $\begin{array}{c}0.959 \\
(1.148)\end{array}$ & & $\begin{array}{c}0.223 \\
(0.899)\end{array}$ & & $\begin{array}{c}-0.609 \\
(1.531)\end{array}$ & & $\begin{array}{c}0.584 \\
(0.857)\end{array}$ & & $\begin{array}{c}-1.258 \\
(1.653)\end{array}$ & & $\begin{array}{c}0.277 \\
(1.456)\end{array}$ & & $\begin{array}{l}-0.007 \\
(0.011)\end{array}$ \\
\hline & $\mathrm{R}^{2}$ & 0.924 & 0.924 & 0.711 & 0.713 & 0.474 & 0.477 & 0.358 & 0.378 & 0.600 & 0.603 & 0.776 & 0.777 & 0.935 & 0.936 & 0.374 & 0.380 \\
\hline & perc_18oumais_fund_comp & Sim & Sim & Sim & Sim & Sim & Sim & Sim & Sim & & & & & & & & \\
\hline & perc_med_comp_18 & Sim & Sim & Sim & Sim & Sim & Sim & Sim & Sim & & & & & & & & \\
\hline & perc_sup_comp_25 & Sim & Sim & Sim & Sim & Sim & Sim & Sim & Sim & & & & & & & & \\
\hline & Água & Sim & Sim & Sim & Sim & Sim & Sim & Sim & Sim & Sim & Sim & Sim & Sim & Sim & Sim & Sim & Sim \\
\hline & Lixo & Sim & Sim & Sim & Sim & Sim & Sim & Sim & Sim & Sim & Sim & Sim & Sim & Sim & Sim & Sim & Sim \\
\hline & Luz & Sim & Sim & Sim & Sim & Sim & Sim & Sim & Sim & Sim & Sim & Sim & Sim & Sim & Sim & Sim & Sim \\
\hline & tx_atividade_18oumais & Sim & Sim & Sim & Sim & Sim & Sim & Sim & Sim & & & & & Sim & Sim & Sim & Sim \\
\hline & Urbano & Sim & Sim & Sim & Sim & Sim & Sim & Sim & Sim & Sim & Sim & Sim & Sim & Sim & Sim & Sim & Sim \\
\hline & tx_analf_18oumais & & & & & & & & & & & & & Sim & Sim & Sim & Sim \\
\hline & tx_envelhecimento & & & & & & & & & & & & & Sim & Sim & Sim & Sim \\
\hline
\end{tabular}

Fonte - Elaborado pelos autores.

Notas - erro padrão entre parênteses. ${ }^{* * *}, * * \mathrm{e}^{*}$ denotam, respectivamente, $\mathrm{p}<0,01, \mathrm{p}<0,05 \mathrm{e} \mathrm{p}<0,1$. 


\section{Considerações finais}

De acordo com a literatura econômica, a expansão universitária por meio da criação de novos campi tende a gerar crescimento e desenvolvimento dos municípios beneficiados. Sendo assim, o presente estudo se propôs a analisar o impacto da expansão das Universidades Federais sobre o desenvolvimento local dos municípios brasileiros, baseado em indicadores econômicos, sociais e de mercado de trabalho; além disso, buscou-se identificar os fatores que influenciaram os municípios a serem contemplados com um campus da Universidade.

Para avaliar o impacto dessa política, optou-se por utilizar o método de pareamento do escore de propensão com objetivo de construir um grupo de controle que mais se assemelhasse ao grupo de tratamento em termos de determinadas características observáveis. E, para estimar os efeitos, após o pareamento, utilizam-se os estimadores de Diferenças em Diferenças e de Efeito Fixo.

Inicialmente, pode-se dizer que municípios que possuem campus universitário particular, população superior a 50 mil habitantes e com maior relevância econômica (importância do PIB) na sua microrregião são fatores (ou características) que influenciaram na escolha dos municípios contemplados com um campus de Universidade Federal por meio da política de expansão.

Em relação aos efeitos da expansão sobre os indicadores, observou-se que a criação de novos campi reduz o percentual de pobres nos municípios, em média, em 1,5 p.p. Além disso, possuem evidencia (fraca) de que aumenta a renda per capita, o percentual de ocupados com nível superior e com nível médio.

Todavia, de uma forma geral, não se observa impactos de curto prazo, visto que os resultados dos modelos de Especificação E2 não foram estatisticamente significantes, a exceção da variável de percentual de ocupados com nível médio. No entanto, vale ressaltar que talvez seja necessária uma defasagem temporal maior para que os efeitos da política de expansão causem impactos significativos sobre a economia, visto que para a Universidade gerar forte impacto no desenvolvimento local, é necessário que os municípios se adequem à nova realidade e consigam absorver os benefícios que a política de expansão das Universidades pode gerar, demandando, algum tempo de maturação. Ademais, há a possibilidade dos campi implantados mais recentemente apresentarem porte muito pequeno, quando comparados ao tamanho da economia local e os investimentos iniciais sejam poucos relevantes para afetar a demanda dos municípios no curto prazo, necessitando, assim, tempo para que os novos campi se consolidem e afetem a demanda de produtos e serviços da economia local. 
Por fim, sugerem-se como pesquisas futuras análises que contemplem informações de oferta e demanda dos cursos nesses novos campi, bem como análises de custo benefício e efetividade dessas políticas de ampliação dos campi universitários federais para aferir se essa política é viável do ponto de vista econômico e também de desenvolvimento regional. Além de pesquisas qualitativas para aferir sobre a percepção da comunidade que teve a instalação desses campis, campus e/ou Universidades.

\section{Referências}

BARBOSA, Marcelo P.; PETTERINI, Francis; FERREIRA, Roberto T. Avaliação do impacto da política de interiorização das universidades federais sobre as economias municipais. In: ENCONTRO DE ECONOMIA DA REGIÃO SUL, 17., 2014, Maringá. Anais [...]. Maringá: UEM, 2014.

BECKER, S. O.; ICHINO, A. Estimation of average treatment effects based on propensity scores. The Stata Journal, EUA, v. 2, n. 4, p. 358-377, 2002.

BATTU, Harminder; FINCH, John. Integrating knowledge effects into university impact studies: a case study of aberdeen university. Aberdeen: Department of Economics University of Aberdeen, 1998.

BOVO, José Murari. A contribuição da Unesp para o dinamismo econômico dos municípios. São Paulo: Ed. UNESP, 2013.

BRASIL. Ministério da Educação. Programa de apoio a planos de reestruturação e expansão das Universidades Federais REUNI 2008 - Relatório de primeiro ano reestruturação e expansão das Universidades Federais. Brasília, DF, 2009.

BRASIL. Ministério da Educação. Expansão da educação superior e profissional e tecnológica: mais formação e oportunidades para os brasileiros. 2011. Disponível em: http://portal.mec.gov.br/expansao/images/APRESENTACAO_EXPANSAO_EDUCACAO_S UPERIOR14.pdf. Acesso em: 1 maio 2017.

BRASIL. Secretaria de Educação Superior. A democratização e expansão da educação superior no país: 2003 - 2014. Brasília, DF, 2015.

CALDARELLI, Carlos E.; CAMARA, Marcia R.; PERDIGÃO, Claudia P. Instituições de ensino superior e desenvolvimento econômico: o caso das universidades estaduais paranaenses. Planejamento e Políticas Públicas, Brasília - DF, v. 44, p. 85-112, 2015.

COCHRAN, William G.; RUBIN, Donald B. Controlling bias in observational studies: a review. The Indian Journal of Statistics, Sankhyã, v. 35, n. 4, p. 417-66, 1973.

FLORAX, Raymond. The university: a regional booster? Economic impacts of academic knowledge infrastructure. Avebury: Aldershot, 1992. 
FOGUEL, Miguel N. Diferenças em Diferenças. In: MENEZES FILHO, N. (org.). Avaliação econômica de projetos sociais. São Paulo: Dinâmica Gráfica e Editora, 2012. p. 69-83.

HOFF, Debora N.; MARTIN, Aline S. S.; SOPEÑA, Mauro B. Universidades e Desenvolvimento Regional: impactos quantitativos da UNIPAMPA em Sant'ana do Livramento. Revista Redes, Santa Cruz do Sul, RS, v.16, n. 3, p.157-183, 2011.

KHANDKER, Shahidur R; KOOLWAL, Gayatri B; SAMAD, Hussain A. Handbook on impact evaluation: quantitative methods and practices. Washington, DC: The World Bank, 2010.

KURESK, Ricardo; ROLIM, Cassio. Impacto econômico de curto prazo das universidades federais na economia brasileira. Revista Paranaense de Desenvolvimento, Curitiba, PR, v. 117, p. 29-51, 2009.

MENEZES FILHO, Naercio (org.). Avaliação econômica de projetos sociais. São Paulo: Dinâmica Gráfica e Editora, 2012.

MORAES, Flavio F. Universidade, inovação e impacto socioeconômico. São Paulo em Perspectivas, São Paulo, SP, v. 14, n. 3, p. 8-11, 2000.

NEVES, Rommel S. Avaliação da interiorização do Instituto Federal de Educação, Ciência e Tecnologia do Maranhão. 2015. 82f. Dissertação (Mestrado) - Programa de Pósgraduação em Economia, Universidade Federal do Ceará, Fortaleza, 2015.

NIQUITO, Thais Waideman; RIBEIRO, Felipe Garcia; PORTUGAL, Marcelo Savino. Impacto da criação das novas universidades federais sobre as economias locais.

Planejamento e Políticas Públicas, Brasília-DF, n. 51, p. 267-394, 2018.

PEIXOTO, Betania. T.; ANDRADE, Monica. V.; AZEVEDO, João P. Avaliação econômica do Programa Fica Vivo: o caso piloto. Belo Horizonte: UFMG/Cedeplar, 2008.

ROLIM, Cassio F. C.; SERRA, Mauricio A. Instituições de ensino superior e desenvolvimento regional: o caso da região Norte do Paraná. Revista de Economia, Curitiba, PR, v. 35, n. 3, p. 87-102, 2009.

ROSENBAUM, Paul R.; RUBIN, Donald B. The central role of the propensity score in observational studies for causal effects. Biometrika, Oxford, v. 70, p. 41-55, 1983.

SCHNEIDER, L. Educação e desenvolvimento: um estudo do impacto econômico da universidade federal no município de Santa Maria (RS). UNIFRA, Santa Maria, 2002.

SIEGFRIED, John J.; SANDERSON, Allen R.; MCHENRY, Peter. The economic impact of colleges and universities. Economics of Education Review, United Kingdom, v. 26, n. 5, p. 546-558, 2007.

SILVA, V. L. O futuro das indústrias e o papel da universidade no desenvolvimento sustentável. Brasília: MDIC, 2001. 
TURNER, David. The economic impact of a university on its environment. European Education, Europe, v. 29, p. 88-95, 1997.

VINHAIS, Henrique Eduardo F. Estudo sobre o impacto da expansão das universidades federais no Brasil. 2013. 234 f. Tese (Doutorado) - Curso de Economia, Universidade de São Paulo, São Paulo, 2013.

WILTGEN, R. S. Notas sobre polarização e desigualdades regionais. Ensaios FEE, Porto Alegre, RS, v. 12, n. 2, p. 532-539, 1991. 